patients with acute exacerbations of COPD. Thorax 2008;63:301-5.

34. Shah BR, Laupacis A, Hux JE, et al. Propensity score methods gave similar results to traditional regression modeling in observational studies: a systematic review. J Clin Epidemiol 2005; 58:550-9.

35. Psaty BM, Koepsell TD, Wagner EH, et al. The relative risk of incident coronary heart disease associated with recently stopping the use of betablockers. JAMA 1990;263:1653-7.

36. Cazzola M, Imperatore F, Salzillo A, et al. Cardiac effects of formoterol and salmeterol in patients suffering from COPD with preexisting cardiac arrhythmias and hypoxemia. Chest 1998;114:411-5.

37. Newhouse MT, Chapman KR, McCallum AL, et al. Cardiovascular safety of high doses of inhaled fenoterol and albuterol in acute severe asthma. Chest 1996;110:595-603.

38. Au DH, Curtis JR, Every NR, et al. Association between inhaled beta-agonists and the risk of unstable angina and myocardial infarction. Chest 2002;121:846-51.

39. Au DH, Lemaitre RN, Curtis JR, et al. The risk of myocardial infarction associated with inhaled betaadrenoceptor agonists. Am J Respir Crit Care Med 2000;161:827-30.

40. Bauer K, Rakusan S, Kaik G. Pulmonary effects of long-term beta 2-blockade in healthy subjects: comparative study of metoprolol OROS. Am Heart J 1990;120:473-7.

41. Bruschi C, Casali L, Cerveri I, et al. Effects of celiprolol on the bronchial reactivity in asthma. Am J Cardiol 1988;61:53-4C.

42. Tattersfield AE, Cragg DJ, Bacon RJ. Assessment of beta-adrenoceptor selectivity of a new betaadrenoceptor antagonist, bisoprolol, in man. $\mathrm{Br} \mathrm{J}$ Clin Pharmacol 1984;18:343-7.

43. Salpeter SR, Ormiston TM, Salpeter EE. Cardioselective beta-blockers in patients with reactive airway disease: a meta-analysis. Ann Intern Med 2002:137:715-25.
44. Everly MJ, Heaton PC, Cluxton RJ Jr. Beta-blocker underuse in secondary prevention of myocardial infarction. Ann Pharmacother 2004;38:286-93.

45. Omenn GS, Goodman GE, Thornquist MD, et al. Effects of a combination of beta carotene and vitamin A on lung cancer and cardiovascular disease. N Engl J Med 1996:334:1150-5.

46. Alpha-Tocopherol, Beta Carotene Cancer Prevention Study Group. The effect of vitamin E and beta carotene on the incidence of lung cancer and other cancers in male smokers. $N$ Engl J Med 1994;330:1029-35.

47. Cardiac Arrhythmia Suppression Trial II Investigators. Effect of the antiarrhythmic agent moricizine on survival after myocardial infarction. N Engl J Med 1992;327:227-33.

48. Cardiac Arrhythmia Suppression Trial (CAST) Investigators. Preliminary report: effect of encainide and flecainide on mortality in a randomized trial of arrhythmia suppression after myocardial infarction. N Engl J Med 1989;321:406-12.

\title{
Overdiagnosis in lung cancer: different perspectives, definitions, implications
}

\section{Peter B Bach}

Does lung cancer screening lead to overdiagnosis? Most lung cancer prevention experts think it does, but there is a contingent who believe that it does not. This latter group holds fast to a notion that essentially all histological foci of lung cancer pose an imminent threat to health, irrespective of how they are discovered. Enter Dr Reich's interesting and thoughtful article, ${ }^{1}$ which provides a cohesive overview of the epidemiological data that would be explained by "overdiagnosis", and therefore the case for its existence (see page 377). Reich also considers the totality of studies that are oft cited as evidence that "overdiagnosis" does not exist. For these articles, he summarises their findings too, and raises his concerns about them. It is an important contribution, that clearly presents Reich's view that overdiagnosis is a serious concern in lung cancer screening. Because it also incorporates opposing evidence, it is a worthy reference for anyone interested in understanding this confusing issue.

My interpretation of the data is much like Reich's-I have little doubt that lung cancer screening, particularly with CT, uncovers vast numbers of lesions with relatively little malignant potential. I also

Correspondence to: Dr Peter B Bach, Memorial SloanKettering Cancer Center, New York, NY 10021, USA; bachp@mskcc.org harbour little doubt that surgical treatment of individuals who are overdiagnosed is potentially very harmful to them, given that surgery confers risks both short and long term. However, as a clinician, I find this epidemiologic concept hard to operationalise because current knowledge does not allow me to distinguish between those histological foci that pose a reduced threat compared with those that pose a very real and imminent threat. Therefore, faced with a positive biopsy at this point, it is essentially an instinctual response to recommend immediate and definitive treatment.

Perhaps this tension between epidemiological data and clinical instinct is what leads to different interpretations of the available data. This would make sense, as my experience is that clinicians and epidemiologists think about the world differently. An alternative is that the tension actually reflects differences in the definition of "overdiagnosis"-so, what appears to be a disagreement is actually a failure of terminology. Irrespective of the reason, whether or not there is a large reservoir of small growths that have limited malignant potential is an important question that affects our ability to interpret single arm studies of screening with survival as an outcome and our ability to manage lesions uncovered by CT screening.

\section{DIFFERENT WEIGHTS APPLIED TO THE SAME DATA}

\section{Contrasting views of falsifying} observations

Treating victim after victim of lung cancer makes it nearly impossible for clinicians to believe that a cluster of lung cancer cells could be harmless. But, it is equally hard for epidemiologists to ignore the findings in virtually every study of lung cancer screening with either chest $x$ ray or CTnamely, that screening uncovers more lung cancers than would otherwise appear sporadically or could conceivably cause illness. Which set of observations you take more seriously depends on your perspective. Clinicians, by the nature of what we do, must view the world in a binary way, because the rubber hits the road each time we make a treatment decision. We must decide to give antibiotics or not to the patient with a pulmonary infiltrate. We do not have the option of reducing the dose of antibiotic so that it reflects a blended probability that pneumonia is present or not. If the patient improves, this makes us more confident that we had the right diagnosis-only if the patient fails to improve do we switch diagnoses. Epidemiologists (many, like Reich, are also clinicians) have as their focus the underlying but unobservable distributions that cause events to happen at a certain frequency. They know that some people with infiltrates have pneumonia and some do not. The rubber hits the road for them when they compare the total number of pneumonias occurring in a population to what their theory predicted. If those numbers are roughly the same, they stick with their theory. When the two do not add up, they switch theories.

This difference between clinical and epidemiologic insights might explain disagreements regarding "overdiagnosis" 
and, more generally, the assumption that all malignant foci of lung cancer will progress to cause illness and death. Clinical experience teaches us that people with lung cancer have very poor prognoses unless they are treated promptly. On the flip side, when we remove small foci of lung cancer, the outcomes are usually quite good. So, clinically, most patients who are fit enough to have their early lung cancers treated get treated-as a result, we never receive information that would contradict our assumptions that small foci of lung cancer would behave in an aggressive manner if left untreated. Epidemiological data, as Reich carefully summarises, overwhelmingly suggests that screening uncovers lung cancers that will not cause harm to patients-therefore, to epidemiologists, there are reasons to switch from the clinical theory that all lung cancers are aggressive to one that accommodates the hypothesis that some (or maybe many) are not.

\section{Different faith in pathologists}

The differences also apply to the interpretation of biopsy results. Clinicians have to determine to treat or not, and in the clinical world, positive histology for cancer is an absolute indication for treatment. The pathologist's interpretation is something we would rarely if ever doubt. This is because the pathologist's interpretation of a biopsy is about the most reliable predictor of the future we have in clinical medicine, and we are obligated when that future looks grim to do something. Epidemiologists have a different perspective. They know that the pathologist cannot actually see into the future based on findings on a slide - the pathologist can only determine if what they see on the slide resembles other clusters of malignant cells that were causing illness in other patients. Also, epidemiologists do not have to make treatment decisions-what they do is distinguish association from causation. It is therefore comfortable to an epidemiologist to look at the finding of histological lung cancer and reason that such a finding is present among tumours that behave in a malignant manner (ie, there is an association), even if such cells can be found in a patient in which there is no malignant clinical precursor (ie, there is no causation).

\section{Different meaning applied to outcomes of untreated populations}

The differences also apply to the interpretation of outcomes occurring among groups of patients who are diagnosed with small foci of lung cancer but are not treated. Reich nicely summarises this literature and raises a mix of clinical and epidemiological concerns about it. Without Reich's guidance, readers may look at these studies and misinterpret them. Specifically, the various studies that report on the survival of untreated patients with clinical stage 1 lung cancer seem to suggest that untreated stage 1 lung cancer is nearly uniformly fatal if untreated. However, a more cautious interpretation is appropriate. Reich, for instance, points out that the studies by Flehinger et al and Henschke et al do not address the question of "overdiagnosis" at all. Each of these studies focuses only on subjects who died of lung cancer. Neither addresses the proportion of subjects who died of a cause other than lung cancer. As Reich points out, the focus only on patients dying of lung cancer is accomplished by "adjustment" in one case and censoring in the other but the effect is the same.

To put a slightly finer point on the matter, consider a cohort of 10 people with clinical stage 1 lung cancer, all untreated. If over the next 5 years nine of these people die of other causes and one dies of lung cancer, one might say that the overdiagnosis rate is $90 \%$ (meaning nine of the 10 cases of lung cancer did not cause harm). Now, if one performs a Kaplan-Meier analysis, and censors all of the individuals who died of another cause, then one would conclude that the death rate from lung cancer is $100 \%$ - a finding that would instead suggest that the overdiagnosis rate is $0 \%$. In other words, the key question in "overdiagnosis" is the overall likelihood that someone diagnosed with lung cancer will not die from it, which can only be addressed if deaths from other causes are not "adjusted for" or censored - it cannot be addressed by examining only the selective likelihood that a person will die of lung cancer if they survive competing causes of death.

Building on Reich's observations, it is worth noting that not only the analyses but the implied comparisons in these studies are problematic. The implied comparison in each case is between an untreated and a treated patient with stage I lung cancer. However, this comparison is guaranteed to find that the survival gap is larger than it really is, and thus create an appearance that untreated early stage lung cancer is more aggressive than it actually is. The bias comes from two main sources. Firstly, treated early stage lung cancer is surgically defined, while untreated early stage lung cancer is usually clinically defined. Clinicians who treated lung cancer patients before there was widespread availability of the fluoro-deoxy-glucose positron emission tomography scan and CT scans will recall that many clinical stage I patients were frequently upstaged during surgery. Thus survival of surgical stage I patients will be superior to that of clinical stage I patients, even given equal treatment. Mountain reported on the magnitude of the gap by stage in lung cancer between clinically and pathologically staged patients, showing that at each stage, survival was markedly better in the pathologically staged group. ${ }^{2}$ Secondly, the treated and untreated groups are not arrived at randomly. Patients not treated for lung cancer are usually too sick from comorbidities, or have other reasons why their physicians believe they are not eligible for aggressive or potentially curative interventions. Therefore, irrespective of the impact of treatment, untreated patients will appear to have worse survival than treated patients for this reason as well.

\section{DIFFERENCES IN DEFINITION}

Reich defines "overdiagnosis" in a clinical context, and the term reflects whether or not there is value to the patient of the diagnosis of lung cancer being made. In this definition, "overdiagnosed" lung cancer is defined as a cancer that will not affect the patient during that patient's lifetime. Such "overdiagnosed" lung cancers would be expected, using such a patient centred definition, to be more common in patients with serious comorbidities, who are likely to die of other causes in the near term. This definition of "overdiagnosis" is the most apt, in my mind, but it is not the only definition. Some have taken the term "overdiagnosis" to be a description of the behaviour of the tumour itself, irrespective of its host. In this context, "overdiagnosed" lung cancer is taken to be synonymous with "benign"3 - a notion that is so foreign to clinicians that they are inclined to reject the whole concept. However, Reich's definition is one that clinicians should find intuitively reasonable - it is not wholly uncommon for clinicians to have particular patients in which the discovery of a small early stage lung cancer is very unlikely to alter their longevity.

A third set of definitions of "overdiagnosis" have been advanced by Henschke and other I-ELCAP investigators, and focus on the appearance or growth rates of the lung cancers, rather than their impact on patients. In a recent analysis, Henschke et al defined "overdiagnosis" as the absence of pathological features consistent with lung cancer in a biopsy positive for lung cancer. ${ }^{4}$ This histological definition supported Henschke et al's conclusion that "overdiagnosis" was rare in CT screening. 
However, it should be noted that the conceptual underpinning of the "overdiagnosis" concept, as outlined by Reich, does not anchor to histological appearance but rather patient experience. In an analogous approach, Yankelevitz et al defined "overdiagnosis" as a phenomenon only present in lung cancers that double in size very slowly. ${ }^{5}$ In applying this radiological definition, Yankelevitz et al were also able to conclude that "overdiagnosis" was rare in the Mayo Lung Project of chest $x$ ray screening. Readers of Reich's article will appreciate the contradiction between Yankelevitz's conclusion and the robust findings from the Mayo Lung Project that documented a strong "overdiagnosis" effect resulting from chest $x$ ray screening. ${ }^{6}$

\section{IMPLICATIONS OF OVERDIAGNOSIS}

If a sizable fraction of small growths found by CT screening pose limited threat to a person's health, Reich notes several important implications. For instance, determining the survival of patients with lung cancer diagnosed by screening will not be a valid way of estimating the benefits of CT detection, as many of the individuals so diagnosed would not have died of lung cancer anyway. In a recent analysis, my colleagues and I illustrated this disconnect. ${ }^{7}$ We found that CT screening led to the discovery of more than three times as many lung cancers as would have been detected sporadically, suggesting a high rate of overdiagnosis. We also observed that the 4 year lung cancer specific survival rate among those with surgical resection was $94 \%$. But we saw that the treatment of these cancers had no measurable impact on the rate of death from lung cancer. We concluded, as did an accompanying editorial, that improvements in survival did not necessarily correlate with a reduction in the lung cancer death rate, most likely because of the high rates of overdiagnosis occurring as a result of regular CT screening. ${ }^{8}$

Competing interests: None.

Thorax 2008;63:298-300. doi:10.1136/thx.2007.082990

\section{REFERENCES}

1. Reich JM. A critical appraisal of overdiagnosis: estimates of its magnitude and implications for lung cancer screening. Thorax 2008;63:377-83.

2. Mountain CF. Revisions in the international system for staging lung cancer. Chest 1997;111:1710-17.

3. Black WC. Overdiagnosis: An underrecognized cause of confusion and harm in cancer screening. J Natl Cancer Inst 2000;92:1280-2.

4. Henschke Cl, Yankelevitz DF, Libby DM, et al. Survival of patients with stage I lung cancer detected on CT screening. N Engl J Med 2006;355:1763-71.

5. Yankelevitz DF, Kostis WJ, Henschke Cl, et al. Overdiagnosis in chest radiographic screening for lung carcinoma: frequency. Cancer 2003;97:1271-5.

6. Bach PB, Kelley MJ, Tate RC, et al. Screening for lung cancer: a review of the current literature. Chest 2003;123(Suppl):72S-82.

7. Bach PB, Jett JR, Pastorino U, et al. Computed tomography screening and lung cancer outcomes. JAMA 2007;297:953-61.

8. Black WC, Baron JA. CT screening for lung cancer: spiraling into confusion? JAMA 2007;297:995-7.

\section{Lung alert}

\section{Industry funded studies for inhaled corticosteroids show greater safety}

There is much debate about the effect on interpretation of results caused by the funding of studies by the pharmaceutical industry. This paper attempts to look for objective differences in results depending on the source of funding for studies on the adverse effects of inhaled corticosteroids.

A Medline search identified original articles on inhaled corticosteroids containing data on adverse effects between 1993 and 2002. The studies were analysed, blinded to funding source, by two authors who categorised side effects and authors' conclusions on drug safety. If there was a discrepancy a third author had a casting vote. Comparison was then made between pharmaceutical industry funded (PF) and non-pharmaceutical industry funded (non-PF) studies.

Non-PF studies were more likely to report significant adverse effects than PF studies. However, the results became non-significant when confounders such as study design were taken into account. However, PF studies were more likely than non-PF studies to conclude a drug was safe when a statistically significant adverse event had occurred in the study. The current authors contacted the authors of non-PF papers (where the authors had not stated a funding source) and, of those who responded, $8.1 \%$ reported pharmaceutical funding and were reallocated in the current study.

This study indicates that authors' conclusions may be influenced by funding sources and reiterates the importance of assessing funding and conflicts of interest when evaluating studies.

- Nieto A, Mazon A, Pamies R, et al. Adverse effects of inhaled corticosteroids in funded and nonfunded studies. Arch Intern Med 2007; 167:2047-53

\section{Patrick Murphy}

Correspondence to: P Murphy, SpR in Respiratory Medicine, Homerton University Hospital, London, UK; p b murphy@hotmail. com 